\title{
Reciprocating Parallel Milling Macro Programming
}

\author{
Mingyao Wang \\ College of Mechanical Engineering, Dalian Vocational Technical College, Dalian, 116037, China \\ 842007536@qq.com
}

Key words: CNC milling machine; reciprocating parallel milling; macro program

\begin{abstract}
The reciprocating parallel milling processing program is written by using the plunge count and plunge height in the $\mathrm{Z}$ direction on CKA6136 CNC milling machine of FANUC0i system and the method and idea of writing the milling open parts surface program by macro programming are analyzed.
\end{abstract}

In the parts processing with $\mathrm{CNC}$ milling machine, the parts are often required to have open surfaces. If there are a small batch and a large variety of parts, the conventional manual programming will cause heavy workload and mistakes easily, so MasterCAM, UG and other software can be used for automatic programming. However, a simple plane may produce hundreds or thousands of programs. Considering this and according to their similarities that require milling open surfaces, the assignments of several variables can be changed for the processing of milling open surfaces of parts with a program if the macro programming is adopted, even if the size of parts to be processed is different, without writing any special program.

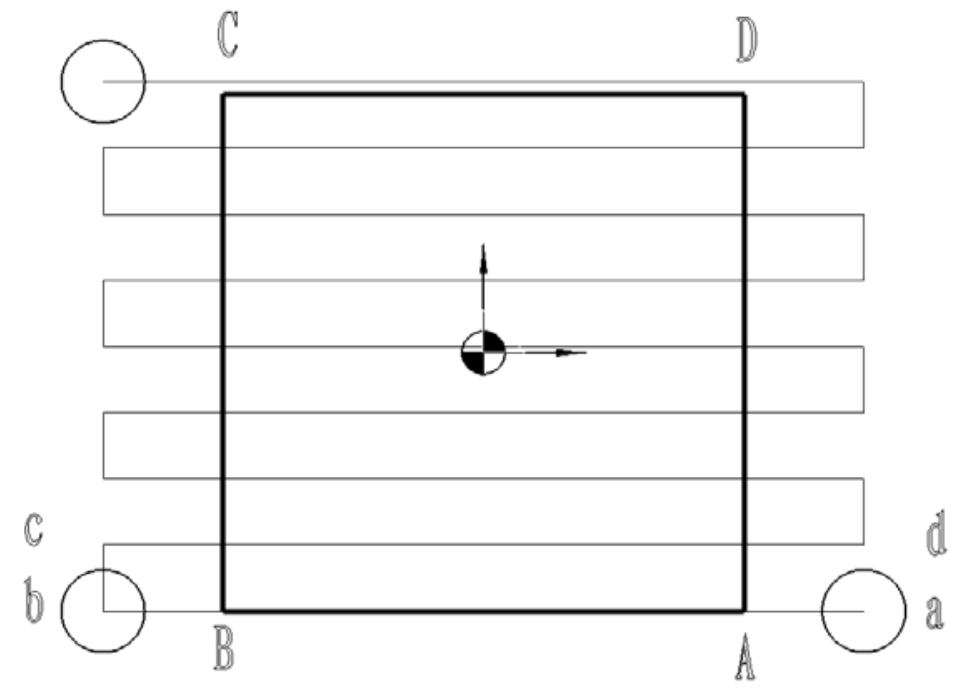

Figure I

The basic idea of programming is to first determine the milling route of a single layer and then determine the changes in height. According to figure I, the surface of parts to be processed is $\mathrm{ABCD}$, the milling cutter moves from $\mathrm{AB}$ side to $\mathrm{CD}$ side parallel according to the route of $\mathrm{a} \rightarrow \mathrm{b} \rightarrow \mathrm{c} \rightarrow \mathrm{d}$ and a reciprocating parallel milling of a single layer can be completed by using the plunge count and plunge height in the $\mathrm{Z}$ direction after single-layer milling to reach the processing depth or height. 


\section{Write reciprocating parallel milling processing program by using the plunge count in the $Z$ direction}

It writes the reciprocating parallel milling processing program by using the plunge count in the $\mathrm{Z}$ direction. The origin of coordinates is the center of parts, the flat-bottomed end mill is adopted and the cutter diameter is $\Phi 16$.

Writing of reciprocating parallel milling program parallel to $\mathrm{X}$-axis:

$\%$

$\mathrm{O} 0001$

G54

G91 G28 Z0

G90 G00 Z100

M03 S800

$\# 1=100$ (Length of rectangle in the $X$ direction: $100 \mathrm{~mm}$ )

$\# 2=100$ (Length of rectangle in the $Y$ direction: $100 \mathrm{~mm}$ )

$\# 3=16$ (Use the flat-bottomed end mill and the cutter diameter is $\Phi 16$ )

$\# 4=-\# 2 / 2$ (Determine the coordinate in the $\mathrm{Y}$ direction according to the assignment of start point and length of rectangle in the $\mathrm{Y}$ direction)

$\# 5=0.8$ (Modify the gradient of independent variable according to the material and the step is 0.8 times)

$\# 7=\# 5^{*} \# 3$ (The step value is 0.8 times of the cutter diameter)

$\# 8=[\# 1+\# 3] / 2+15$ (Determine the coordinate of milling start point of rectangle in the $\mathrm{X}$ direction, 15 is the safe distance of displacement)

\#9=0 (Processing count in the $Z$ direction, the initial assignment is 0 )

$\# 10=10$ (Total removal rate in the $Z$ direction)

$\# 11=4$ (Working allowance in the $Z$ direction is removed by 4 times)

$\# 12=\# 10 / \# 11$ (Determine the cutting depth according to the total amount and count)

$\# 13=0$ (Coordinate of parts in the $\mathrm{Z}$ direction)

N1000 G00 X\#8 Y\#4 F200

$\# 9=\# 9+1$ (Determine the start point of $\mathrm{X}$-axis of reciprocating milling, the cycle count in the $\mathrm{Z}$ direction plus 1 for each time)

$\# 13=\# 13+\# 12$ (Determine the coordinate of circulatory cutting in the $\mathrm{Z}$ direction)

G01 Z-\#13 (Straightly feed to the cutting depth)

\#14=\#4 (Determine the start point coordinate of Y-axis of reciprocating milling)

N2000 G01 X\#8 (Process from the right side to the left side of workpiece parallel )

$\# 14=\# 14+\# 7$ (Determine the step value)

Y\#14 (Process on the left side of parts parallel to Y-axis)

$\mathrm{X} \# 8$ (Process from the left side to the right side of workpiece parallel )

\#14=\#14+\#7 (Determine the step value)

Y\#14 (Process on the right side of parts parallel to $\mathrm{Y}$-axis)

I F [\#14 L T[\#2/2+0.2*\#3]] GOTO2000 (Conditional transfer statement, conditional judgment of processing location at $\mathrm{Y}$-axis

G0 Z30 (Lift the cutter to the safe location after parallel milling)

I F [\#9 L T\#11] GOTO1000 (Conditional transfer statement, conditional judgment of count in the $\mathrm{Z}$ direction)

G0 Z200

M05 
M30

$\%$

\section{Write reciprocating parallel milling processing program by using the plunge height in the $Z$} direction

It writes the reciprocating parallel milling processing program by using the plunge height in the $\mathrm{Z}$ direction. The origin of coordinates is the center of parts, the flat-bottomed end mill is adopted and the cutter diameter is $\Phi 16$.

$\%$

$\mathrm{O} 0002$

G54

G91 G28 Z0

G90 G00 Z100

M03 S800

$\# 1=100$ (Length of rectangle in the $\mathrm{X}$ direction: $100 \mathrm{~mm}$ )

$\# 2=100$ (Length of rectangle in the Y direction: $100 \mathrm{~mm}$ )

$\# 3=16$ (Use the flat-bottomed end mill and the cutter diameter is $\Phi 16$ )

$\# 4=-\# 2 / 2$ (Determine the coordinate in the $\mathrm{Y}$ direction according to the assignment of start point and length of rectangle in the $\mathrm{Y}$ direction)

$\# 5=0.8$ (Modify the gradient of independent variable according to the material and the step is 0.8 times)

$\# 7=\# 5^{*} \# 3$ (The step value is 0.8 times of the cutter diameter)

$\# 8=[\# 1+\# 3] / 2+15$ (Determine the coordinate of milling start point of rectangle in the $\mathrm{X}$ direction, 15 is the safe distance of displacement)

$\# 9=0$ (Coordinate assignment in the $\mathrm{Z}$ direction, the initial value is 0 )

$\# 10=30$ (Total removal rate in the $Z$ direction)

$\# 11=5$ (Removal height in the $Z$ direction)

N3000 G00 X\#8 Y\#4 F200 (Determine the start point of X-axis of reciprocating milling)

$\# 9=\# 9+\# 11$ (Processing height assignment in the $\mathrm{Z}$ direction)

Z-\#9F200 (Height from plunge to assignment in the $\mathrm{Z}$ direction)

\#14=\#4 (Determine the start point of Y-axis of reciprocating milling)

N2000 G01 X\#8 (Process from the right side to the left side of workpiece parallel )

$\# 14=\# 14+\# 7$ (Determine the step value)

Y\#14 (Process on the left side of parts parallel to Y-axis)

$\mathrm{X} \# 8$ (Process from the left side to the right side of workpiece parallel )

$\# 14=\# 14+\# 7$ (Determine the step value)

Y\#14 (Process on the right side of parts parallel to Y-axis)

I F [\#14 L T[\#2/2+0.2*\#3]] GOTO3000 (Conditional transfer statement, conditional judgment of processing location at $\mathrm{Y}$-axis

G0 Z30 (Lift the cutter to the safe location after parallel milling)

I F [\#9 L T\#10] GOTO4000 (Conditional transfer statement, judgment of processing height in the $\mathrm{Z}$ direction)

G0 Z200

M05

M30

$\%$ 


\section{Conclusion}

The programming should first determine the independent variable and then determine the plunge direction whether it is parallel to $\mathrm{X}$-axis or $\mathrm{Y}$-axis according to the milling direction of parts and finally conduct conditional judgment of end point by using the conditional transfer statement. During parallel reciprocating milling, the step determines the processing speed. If the step is small, there will be more operations of CNC system and plunge counts, thus the processing efficiency is low and the precision is high; if the step is large, there will be less operations of CNC system and plunge counts, thus the processing efficiency is high and the precision is low. Therefore, the step must be chosen reasonably according to the processing requirements. Generally, a larger step is chosen on the premise of meeting the processing requirements. The single-layer processing is completed by using the plunge count or plunge height in the $\mathrm{Z}$ direction. In addition, attention should be paid to the location of the origin of coordinate of program during programming and tool setting.

\section{References}

[1]Peter Smid, CNC Programming Handbook. Beijing: Chemical industry Press, 2005.

[2]Shen Jianfeng, Yujun. Numerical Control Lathe Worker( advanced). Beijing: Engineering Industry Press, 2006.

[3]Zhou Baoniu. CNC Programming and Machining Technology. Beijing: Engineering Industry Press, 2009. 